\section{Transumbilical Single-hole Gasless Ventral Laparoscopic Appendectomy on Elderly Patients with Acute Appen- dicitis}

Sir,

Acute appendicitis is a common clinical and frequent acute abdomen. ${ }^{1}$ Elderly patients with acute appendicitis are often diagnosed with cardiopulmonary dysfunction and liver and kidney dysfunction, which are not able to be tolerated when pneumoperitoneum and general anesthesia put into application. Laparoscopy without $\mathrm{CO}_{2}$ pneumoperitoneum can avoid adverse effects of $\mathrm{CO}_{2}$ on the human body. ${ }^{2}$ The continuous epidural anesthesia is applied to the gasless method. It can also avoid the damage of cardiopulmonary and liver and kidney function caused by general anesthesia. There is no need to worry about the blood gas changes, caused by long operation time, which greatly increase the safety coefficient of elderly patients. It is not easy to observe the scar after healing, so that the aesthetics is further strengthened. It not only enhances the appearance, but also reduces the damage, shortens the postoperative recovery time, and rehabilitates more smoothly. ${ }^{3}$ The objective of this study was to observe the efficacy of transumbilical single-hole gasless ventral laparoscopic appendectomy in the treatment of elderly patients with acute appendicitis.

Sixty-five patients with clinically diagnosed acute appendicitis were selected as subjects. Exclusion criteria were those patients who underwent abdominal color Doppler ultrasound or CT which revealed that there was a mass in the right lower quadrant. Patients with appendicitis could not be diagnosed clearly, patients with onset time more than 36 hours, patients with malignant tumors, diabetes and psychosis, patients with history of abdominal surgery. Patient's skin was prepared before operation. The patients received intramuscular injection of $10 \mathrm{mg}$ diazepam and emptied the bladder before surgery. Continuous and epidural anesthesia was used.

Transumbilical single-hole gasless ventral laparoscopic appendectomy was performed in the supine position with legs spread. The surgeon and his assistant stood on the left side of the patient, while the monitor was on the right side of the patient. A $1.5-2.0 \mathrm{~cm}$ arc incision was made in umbilical region and extended to abdominal cavity layer upon layer. An incision protector was placed into it. Along the extension line of McBurney incision in the lower right abdomen, a K-wire was penetrated up and down into the skin, travelled subcutaneously and then driven out. The subcutaneous travel distance was about $15.0 \mathrm{~cm}$. After both ends of the K-wire was fixed onto a traction bow, the bow was elevated using a hanging rod and a cross bar, so that the wall of the lower right abdomen could be lifted, thereby building an operating space. A laparoscope was placed in the space via the umbilical incision, so that the surgeon could probe the abdominal cavity. Moreover, a harmonic scalpel and a grasping forceps were placed beside the laparoscope in the space via the same incision. With the forceps in the left hand and the scalpel in the right hand, the surgeon moved the greater omentum of the lower right abdomen away, lifted the cecum, searched for the appendix along the taeniae coli, observed the inflammation degree and adhesion of appendix and treated the mesoappendix till the appendix base, using the scalpel. The appendix base was closed with a titanium double-shanked clip or secured with a hem-o-lok clip or endoloop ligature. After that, the appendix was cut off at $5.0 \mathrm{~mm}$ on the far side of the ligation line. Theappendixstump was treated by means of electrocoagulation, without the need of embedding. The appendix was taken out directly via the umbilical region. When no side injury was found in the second probe, the laparoscope and operating equipment were withdrawn, the incision protector was removed, and the umbilical incision was sutured layer upon layer. All patients were treated with the same group of experienced doctors.

Table I: Descriptive statistics of all patients undergoing transumbilical single-hole laparoscopy.

\begin{tabular}{|l|l|}
\hline Variable & Number \\
\hline Age range (years) & $61-78$ \\
\hline Mean age (years) & $69.52 \pm 2.76$ \\
\hline Gender & $38(58.46 \%)$ \\
\hline Male & $27(41.54 \%)$ \\
\hline Female & $25(38.46 \%)$ \\
\hline Comorbidity & $20(30.77 \%)$ \\
\hline Hypertension & $9(13.85 \%)$ \\
\hline Coronary heart disease and cardiac dysfunction & $25-90$ \\
\hline Chronic obstructive pulmonary disease & $36.84 \pm 4.51$ \\
\hline Operation time range (minutes) & $36(55.38 \%)$ \\
\hline Mean operation time (minutes) & $24(36.92 \%)$ \\
\hline Postoperative pathological results & $5(7.69 \%)$ \\
\hline Appendicitis alone & $4-30$ \\
\hline Suppurative appendicitis & $19.62 \pm 2.17$ \\
\hline Gangrenous perforated appendicitis & $2-5$ \\
\hline Postoperative anal exhaust time range (hours) & $4.14 \pm 0.52$ \\
\hline Mean postoperative anal exhaust time (hours) \\
\hline Hospital stay range (days) & \\
\hline Mean hospital stay (days) &
\end{tabular}

Among 65 patients, 38 cases (58.46\%) were males and 27 cases $(41.54 \%)$ were females, aged 61-78 $(69.52 \pm 2.76)$ years. Comorbidities included 25 cases $(38.46 \%)$ of hypertension, 20 cases $(30.77 \%)$ of coronary heart disease and cardiac dysfunction, and 9 cases (13.85\%) of chronic obstructive pulmonary disease (Table I). All the 65 patients underwent the surgery successfully, with an operation time of 25-90 (36.84 \pm 4.51$)$ minutes (Table I). Thirty-six cases $(55.38 \%)$ were diagnosed with appendicitis alone, 24 cases (36.92\%) were infectious with suppurative appendicitis, and 5 cases (7.69\%) suffering from gangrenous perforated appendicitis were confirmed by the postoperative pathological results. Seventeen patients $(26.15 \%)$ were placed with drainage tube after operation without infection of drainage orifice. No patient was used anal- 
gesics after surgery. Postoperative anal exhaust time was 4-30 $(19.62 \pm 2.17)$ hours and hospital stay was 2-5 (4.14 \pm 0.52$)$ days. All patients were followed upforoneto 12 months. Complications such as wound infection, puncture, recurrent right lower abdominal pain, and adhesive intestinal obstruction were not observed in any of them.

The results of this study showed that transumbilical single-hole ventral laparoscopic appendectomy is simple, safe, with fewer complications and quick recovery, and is suitable for elderly patients. This conclusion is basically consistent with the findings of Munakata et al. ${ }^{4}$ However, the transumbilical single-hole gasless ventral laparoscopic appendectomy has some limitations. For example, the suspension rod and the cross bar of the suspension device may affect the operation of the operator, and it is often necessary to insert two or more instruments through the same incision during surgery. Meanwhile, the surgeon and assistant also have conflicts in the station; the obstruction of the abdominal wall, after obsessive abdominal wall suspension, increases difficulty in surgery. ${ }^{5}$ The solution to the above problem is to expand the operating space, use extended instruments and laparoscopes to use angled or articulated instruments and use more small-diameter instruments during surgery, so that more operating space could be obtained. The author believed that with the continuous updating and improvement of the instruments and the regular accumulation of surgical experience, transumbilical single-hole ventral laparoscopic appendectomy can provide a safer protection to elderly patients with acute appendicitis who cannot tolerate pneumoperitoneum.

\section{CONFLICT OF INTEREST:}

Authors declared no conflict of interest.

\section{AUTHORS' CONTRIBUTION:}

ZQ, XL: Did the acquisition, analysis, and interpretation of data for the work; drafted and revised the manuscript critically for the intellectual content; and gave final approval of the version to be published.

\section{REFERENCES}

1. Buhamed F, Edward M, Shuaib A. Synchronous acute appendicitis and acute cholecystitis: Is it a myth or reality? A literature review. Open Access Emerg Med 2019; 11:201-3.

2. Rademaker BMP, Meyer DW, Bannenberg JJG, Klopper PJ, Kalkman CJ. Laparoscopy without pneumoperitoneum. Surgical Endoscopy 1995; 9: 797-801.

3. Canes D, Desai MM, Aron M, Haber GP, Goel RK, Stein RJ, et al. Transumbilical single-port surgery evolution and current status. European Urology 2008; 54(5):1020-9.

4. Munakata K, Uemura M, Shimizu J, Miyake M, Hata T, Ikeda $K$, et al. Gasless transumbilical laparoscopic-assisted appendectomy as a safe and cost-effective alternative surgical procedure for mild acute appendicitis. Surg Today 2015; 46(3):319-25.

5. Kim HO, Chang HY, Lee SR, Son BH, Yong LP, Shin JH, et al. Pain after laparoscopic appendectomy: A comparison of transumbilical single-port and conventional laparoscopic surgery. J Korean Surg Soc 2012; 82(3): 172-8.

Zhigang Qiu and Xichun Liu

Department of Gastrointestinal Surgery, The Affiliated Hospital of Qingdao University, Qingdao, Shandong, China

Correspondence to: Xichun Liu, Department of Gastrointestinal Surgery, The Affiliated Hospital of Qingdao University, Qingdao 266000, Shandong, China

E-mail: xhce97@163.com

Received: September 30, 2019; Revised: November 12, 2019; Accepted: December 26, 2019

DOI: https://doi.org/10.29271/jcpsp.2020.09.995 